\title{
Avaliação do coeficiente de variação experimental para caracteres de frutos de pimenteiras
}

\author{
Anderson Rodrigo da Silva ${ }^{*}$, Paulo Roberto Cecon ${ }^{2}$, Elizanilda Ramalho do Rêgo ${ }^{3}$, Moysés Nascimento $^{4}$
}

\section{RESUMO}

O objetivo deste estudo foi formular uma classificação para coeficientes de variação, de experimentos com pimentas do gênero Capsicum, para utilização em seis variáveis morfológicas do fruto. Foram selecionados 38 experimentos com resultados publicados, em que se constaram dados de caracterizações morfológicas do fruto e coeficientes de variação (CV) das seguintes variáveis respostas: peso médio do fruto, comprimento do fruto, comprimento do pedúnculo, maior e menor diâmetro do fruto e espessura do pericarpo. As faixas de classificação dos CV foram baseadas na metodologia proposta por Garcia (1989), que considera a propriedade da distribuição normal do CV. Pelo teste de Lilliefors, a 5\% de probabilidade, todas as variáveis satisfizeram a pressuposição de distribuição normal do CV. As faixas de classificação do CV, obtidas para essas seis variáveis, foram distintas daquelas da classificação geral, proposta por Gomes (2000). Ademais, constatou-se que as classificações dos coeficientes de variação de variáveis morfológicas de pimenteiras do gênero Capsicum dependem da variável resposta, sendo que as maiores faixas de classificação de CV ocorreram para menor diâmetro do fruto, espessura do pericarpo e peso médio do fruto.

Palavras-chave: Capsicum spp., classificação de CV, pimentas, experimentos.

\section{ABSTRACT}

\section{Evaluation of the experimental coefficient of variation of pepper traits}

The aim of this study was to formulate a classification for coefficients of variation of experiments with Capsicum to be used in six morphological fruit variables. We selected 38 trials with published results using data on morphological characterization of fruit and coefficients of variation $(\mathrm{CV})$ of the following response variables: average fruit weight, fruit length, peduncle length, smallest and largest fruit diameter and pericarp thickness. The CV classification ranges were based on the methodology proposed by Garcia (1989), which considers the property of the normal distribution of $\mathrm{CV}$. All variables met the assumption of normal distribution of $\mathrm{CV}$ by the Lilliefors test at $5 \%$ probability. The CV classification ranges obtained for these six variables were distinct from those of the general classification proposed by Gomes (2000). Moreover, it was found that ratings of the coefficients of variation of morphological variables of pepper belonging to the genus Capsicum depend on the response variable, and the largest CV classification ranges were for smaller fruit diameter, pericarp thickness and average fruit weight.

Key words: Capsicum spp., CV classification, pepper, experiments.

\footnotetext{
Recebido para publicação em junho de 2010 e aprovado em janeiro de 2011

${ }^{1}$ Engenheiro-Agrônomo. Mestrando em Estatística Aplicada e Biometria, Universidade Federal de Viçosa. Av. P.H. Rolfs, s/n, 36570-000, Viçosa, Minas Gerais, Brasil. anderson.rodrigo@ufv.br

2 Engenheiro-Agrônomo, Doutor. Universidade Federal de Viçosa. Av. P.H. Rolfs, s/n, 36570-000, Viçosa, Minas Gerais, Brasil. cecon@ufv.br

Bacharel em Biologia, Doutora. Universidade Federal da Paraíba, 59397-000, Areia, Paraíba, Brasil. elizanilda@cca.ufpb.br.

Bacharel em Estatística, Doutor. Universidade Federal de Viçosa. Av. P.H. Rolfs, s/n, 36570-000, Viçosa, Minas Gerais, Brasil. moysesnascim@ufv.br
} 


\section{INTRODUÇÃO}

A pimenta (Capsicum spp.) é uma cultura importante, no Brasil, e tem usos variados como hortaliça e condimento, ou, como planta medicinal e ornamental. $\mathrm{O}$ aumento da produtividade no cultivo desta hortaliça requer trabalhos de melhoramento vegetal e técnicas de experimentação (Rêgo et al. 2009; 2010; Sapucay et al., 2009.

Quanto às técnicas experimentais, é sabido que resultados mais precisos e informações confiáveis são obtidos quando se reduz o efeito do erro experimental. Este, por sua vez, é causado por vários fatores que conduzem à heterogeneidade do material experimental como, por exemplo, a competição intra e interparcelar e a heterogeneidade do solo (Storck et al., 2000), bem como as anormalidades edáficas, agravadas em regiões sob efeito de clima tropical (Ramalho, 2000).

$\mathrm{Na}$ experimentação agrícola, é comum que pesquisadores procurem obter medidas que avaliem a precisão do experimento. A pesquisa é fundamental para a melhoria da produtividade, sendo que a avaliação da qualidade do experimento, usualmente, passa pela verificação do indicador da precisão, expressa pelos valores dos coeficientes de variação (CV) ou da diferença mínima significativa (dms). Conforme Ferreira (1991), o CV é uma medida de variabilidade que mede percentualmente a relação entre $o$ desvio padrão (s) e a média aritmética, com a vantagem de ser um valor adimensional, independentemente da unidade de medida utilizada. A publicação desses indicadores da precisão, obtidos com base no maior número de experimentos semelhantes possíveis, torna-se necessária para a avaliação da qualidade da pesquisa. Snedecor \& Cochran (1980) e Storck et al. (2000) relataram que a distribuição dos valores de $\mathrm{CV}$ possibilita estabelecer faixas que orientem o pesquisador sobre a precisão do experimento.

Segundo Gomes (2000), o coeficiente de variação dá uma ideia da precisão do experimento. O mesmo autor considerou a seguinte classificação para os coeficientes de variação, obtidos em experimentos agrícolas de campo: baixo, quando inferiores a $10 \%$; médios, de 10 a $20 \%$; altos, de 20 a 30\%; e muito altos, quando superiores a $30 \%$. Não obstante, Ferreira (1991) propôs classificá-los para expressar a precisão experimental, da seguinte forma: $\mathrm{CV} \leq 10 \%$ - ótima precisão; $10<\mathrm{CV} \leq 15 \%$ - boa precisão; $15<\mathrm{CV} \leq 20 \%$ - precisão regular; $20<\mathrm{CV} \leq 30 \%$ - péssima precisão experimental; CV > 30\% - precisão “muito péssima". Segundo Campos (1984), o coeficiente de variação nos ensaios agrícolas de campo é esperado entre $10 \mathrm{e} 20 \%$.

Essas classificações de CV, apesar de terem bastante utilidade nas discussões de resultados da pesquisa agrícola em geral, são impróprias por não levarem em conta que a avaliação da precisão depende da variável resposta sob consideração. Em particular, uma classificação de CV apropriada para caracteres como tamanho dos frutos de pimenta deve considerar a espécie, a característica avaliada, o número de repetições, o delineamento experimental, entre outros aspectos importantes.

Esse fato foi constatado por Garcia (1989), que, em experimentos com Eucalipto e Pinus, propôs classificar o CV de uma determinada característica segundo critério que se baseia na média e no desvio padrão dos valores de CV, observados em experimentos semelhantes, pressupondo distribuição normal. Esse critério de classificação foi também utilizado em milho (Scapim et al., 1995), soja (Carvalho et al., 2003) e meloeiro (Lima et al., 2004). Quando os CV não satisfazem a pressuposição de distribuição normal, Costa et al. (2002) sugeriram a utilização das estatísticas mediana e pseudo-sigma, em substituição à média e ao desvio padrão, respectivamente. Estes mesmos autores afirmaram, ainda, que, quando há normalidade, essas duas metodologias fornecem classificações equivalentes.

O objetivo foi obter uma classificação para coeficientes de variação de experimentos com pimentas do gênero Capsicum, para seis variáveis morfológicas do fruto.

\section{MATERIAL E MÉTODOS}

Foram utilizados dados de 38 experimentos, selecionados de trabalhos publicados, desenvolvidos no Laboratório de Biotecnologia e Melhoramento de Hortaliças, Centro de Ciências Agrárias da Universidade Federal da Paraíba, no período de junho de 2007 a dezembro de 2009 . Os experimentos foram todos conduzidos com delineamento de blocos completos ao acaso. Os dados utilizados referem-se a seis características morfológicas do fruto, de nove acessos (variedades), de quatro espécies do gênero Capsicum. As espécies avaliadas foram: Capsicum chinense, $C$. annuит, $C$. baccatum e $C$. frutescens. Foram consideradas as seguintes características: peso médio do fruto, em gramas (PMF); comprimento do pedúnculo (CP); comprimento do fruto $(\mathrm{CF})$; maior diâmetro do fruto (MADF); menor diâmetro do fruto (MEDF) e espessura do pericarpo (EP), em milímetros, medidos com um paquímetro digital, modelo Stainless Hardened, precisão $0,01 \mathrm{~mm}$. O peso médio do fruto, em gramas, foi determinado por meio de pesagem em balança eletrônica Bioprecisa, modelo JA3003N.

As faixas de classificação do coeficiente de variação, para cada uma dessas variáveis, foram elaboradas com base na metodologia proposta por Garcia (1989), que utiliza a média e o desvio padrão (s) dos coeficientes de variação, com os critérios de definição dos limites de classes descritos na Tabela 1.

Inicialmente, foram calculados os valores médios de CV (\%) para cada variável em estudo, determinando os seus respectivos desvios padrão. Os valores de CV foram 
submetidos ao teste de normalidade proposto por Lilliefors (1967), a 5\% de probabilidade, em cumprimento da exigência em cumprimento da exigência de distribuição normal do CV pelo método proposto por Garcia (1989). Os procedimentos estatísticos foram realizados com o auxílio do programa GENES, versão 2006, 4.1 (Cruz, 2006).

\section{RESULTADOS E DISCUSSÃO}

De acordo com a Tabela 2, a pressuposição de distribuição normal do coeficiente de variação é aceita para todas as variáveis em estudo. Portanto, espera-se que a maioria dos CV encontrados para estas variáveis em experimentos situe-se em torno do valor médio, que, neste caso, segundo a classificação de Gomes (2000), mostrou-se "médio" para todas as variáveis, com exceção do maior diâmetro do fruto (MADF), que apresentou classificação "baixo".

Na Tabela 3, encontram-se as classificações propostas para as variáveis morfológicas de frutos do gênero Capsicum analisadas. Nota-se que as variáveis apresentaram faixas de classificação de CV diferentes da classi-

Tabela 1. Critérios de classificação para o coeficiente de variação (CV), propostos por Garcia (1989), com base na média e desvio padrão (s) dos valores de CV

\begin{tabular}{lc}
\hline Critério & Classificação \\
\hline Valores menores que CV médio $-1 \mathrm{~s}$ & Baixo \\
Valores entre CV médio $+/-1 \mathrm{~s}$ & Médio \\
Valores entre CV médio $+1 \mathrm{~s} \mathrm{e} \mathrm{CV} \mathrm{médio}+2 \mathrm{~s}$ & Alto \\
Valores maiores que CV médio $+2 \mathrm{~s}$ & Muito alto \\
\hline
\end{tabular}

ficação geral de CV, proposta por Gomes (2000). Esses resultados são semelhantes aos encontrados por Ledo et al. (2003), quando estudaram o coeficiente de variação de diversas variáveis de bananeira, verificando discordância entre as classificações obtidas e a classificação geral. Os mesmos resultados foram encontrados por Mohallem et al. (2008), ao estudarem coeficientes de variação em experimentos com frangos de corte, verificando que as faixas de classificação dos CV para esses experimentos têm características próprias e diferem das classificações gerais, fazendo-se necessário uma classificação específica, que considere a natureza das variáveis em consideração. Este fato também foi verificado por Amaral et al. (1997).

Verifica-se, ainda, que em uma mesma classificação, houve diferenças nos valores de CV. Por exemplo, entende-se que um coeficiente de variação experimental para a variável comprimento do fruto (CF) é considerado muito alto, quando superior a $17,90 \%$, mas, quando se trata do menor diâmetro do fruto (MEDF), consideramos CV muito alto quando superior a $29,98 \%$, que coincide com a classificação geral proposta por Gomes (2000). Isso pode ser explicado pelo fato de a variável MEDF ser, naturalmente, de maior variabilidade entre frutos da mesma planta ou, mesmo, pelo fato de esta ser uma característica de medições pouco homogêneas, como não o é quando se mede o comprimento do fruto, por exemplo. Ledo et al. (2003), avaliando CV de experimentos com bananeira, também verificaram maiores valores na variável diâmetro do pseudocaule.

Tabela 2. Número de dados (N), média, desvio padrão e teste de Lilliefors para dados de coeficientes de variação (\%), obtidos de variáveis morfológicas de frutos em experimentos com pimenteiras (capsicum spp.)

\begin{tabular}{lcccc}
\hline Variável & N & Média & Desvio padrão & Lilliefors \\
\hline PMF & 38 & 18,01 & 5,79 & $0,1174^{\text {NS }}$ \\
CP & 38 & 11,53 & 3,04 & $0,0955^{\text {NS }}$ \\
CF & 38 & 10,12 & 3,89 & $0,1437^{\text {NS }}$ \\
MADF & 38 & 9,12 & 2,51 & $0,1256^{\text {NS }}$ \\
MEDF & 38 & 19,14 & 5,42 & $0,1212^{\text {NS }}$ \\
EP & 38 & 18,51 & 6,22 & $0,1486^{\text {NS }}$ \\
\hline
\end{tabular}

$\mathrm{NS}=$ não significativo pelo teste de Lilliefors a $5 \%$ de probabilidade; $\mathrm{PMF}=$ peso médio dos frutos; $\mathrm{CP}=$ comprimento do pedúnculo; $\mathrm{CF}=$ comprimento do fruto; $\mathrm{MADF}$ e MEDF = maior e menor diâmetro do fruto, respectivamente; $\mathrm{EP}=$ espessura do pericarpo.

Tabela 3. Classificação dos coeficientes de variação (CV\%) de pimenteiras Capsicum spp. para variáveis morfológicas de frutos: peso médio do fruto (PMF), comprimento do pedúnculo (CP), comprimento do fruto (CF), maior e menor diâmetro do fruto (MADF e MEDF, respectivamente) e espessura do pericarpo (EP)

\begin{tabular}{lcccccc}
\hline Variável & $\mathbf{C V}(\boldsymbol{\%})$ & $\mathbf{S}(\boldsymbol{\%})$ & Baixo & Médio & Alto & Muito alto \\
\hline $\mathrm{PMF}$ & 18,01 & 5,79 & $\mathrm{CV} \leq 12,22$ & $12,22<\mathrm{CV} \leq 23,80$ & $23,80<\mathrm{CV} \leq 29,59$ & $\mathrm{CV}>29,59$ \\
$\mathrm{CP}$ & 11,53 & 3,04 & $\mathrm{CV} \leq 8,49$ & $8,49<\mathrm{CV} \leq 14,57$ & $14,57<\mathrm{CV} \leq 17,61$ & $\mathrm{CV}>17,61$ \\
$\mathrm{CF}$ & 10,12 & 3,89 & $\mathrm{CV} \leq 8,23$ & $8,23<\mathrm{CV} \leq 14,01$ & $14,01<\mathrm{CV} \leq 17,90$ & $\mathrm{CV}>17,90$ \\
$\mathrm{MADF}$ & 9,12 & 2,51 & $\mathrm{CV} \leq 6,61$ & $6,61<\mathrm{CV} \leq 11,63$ & $11,63<\mathrm{CV} \leq 14,14$ & $\mathrm{CV}>14,14$ \\
$\mathrm{MEDF}$ & 19,14 & 5,42 & $\mathrm{CV} \leq 13,72$ & $13,72<\mathrm{CV} \leq 24,56$ & $24,56<\mathrm{CV} \leq 29,98$ & $\mathrm{CV}>29,98$ \\
$\mathrm{EP}$ & 18,51 & 6,22 & $\mathrm{CV} \leq 12,29$ & $12,29<\mathrm{CV} \leq 24,73$ & $24,73<\mathrm{CV} \leq 30,95$ & $\mathrm{CV}>30,95$ \\
\hline
\end{tabular}

Rev. Ceres, Viçosa, v. 58, n.2, p. 168-171, mar/abr, 2011 
As variáveis que apresentaram classes de $\mathrm{CV}$ mais elevadas foram: menor diâmetro do fruto (MEDF), espessura do pericarpo (EP) e peso médio do fruto (PMF), sendo que o maior desvio padrão foi observado na variável EP, o que significa maior amplitude das faixas de classificação. Isso pode ser devido ao formato pouco uniforme dos frutos, o que incorre em alta variabilidade das medidas e, como consequência, altos valores de CV e desvio padrão.

As classificações de CV que mais se aproximaram da classificação proposta por Gomes (2000) foram as das variáveis PMF, MEDF e EP. As demais (CP, CF e MADF) apresentaram classificações inferiores.

\section{CONCLUSÕES}

A classificação dos coeficientes de variação de variáveis morfológicas de pimenteiras do gênero Capsicum depende da variável em estudo.

O método proposto por Garcia (1989) permitiu uma classificação mais adequada dos coeficientes de variação, confirmando que a variabilidade decorrente do erro experimental depende da variável resposta e que, portanto, o critério de avaliação da precisão experimental de ensaios com pimenteiras com base no CV deve ser específico para cada variável.

\section{REFERÊNCIAS}

Amaral AM, Muniz JA \& Souza M (1997) Avaliação do coeficiente de variação como medida da precisão na experimentação com citros. Pesquisa Agropecuária Brasileira, Brasília, 32:1221-1225.

Campos H (1984) Estatística aplicada à experimentação com canade-açúcar. Piracicaba, FEALQ. 292p.

Carvalho CGP, Arias CAA, Toledo JFF, Almeida LA, Kiihl RAS, Oliveira MF, Hiromoto DM \& Takeda C (2003) Proposta de classificação dos coeficientes de variação em relação à produtividade e altura da planta de soja. Pesquisa Agropecuária Brasileira, 38:187-193.

Costa NHAD, Seraphin JC \& Zimmermann FJP (2002) Novo método de classificação de coeficientes de variação para a cultura do arroz de terras altas. Pesquisa Agropecuária Brasileira, 37:243-249.

Cruz CD (2006) Programa Genes versão Windows. Aplicativo Computacional em Genética e Estatística. Viçosa, UFV. 648p.

Ferreira PV (1991) Estatística experimental aplicada à agronomia. Maceió, EDUFAL. 437p.

Garcia CH (1989) Tabelas para classificação do coeficiente de variação. Piracicaba, IPEF. 12p. (Circular técnica, 171).

Gomes FP (2000) Curso de estatística experimental. $14^{\mathrm{a}}$ ed. Piracicaba, Degaspari. 477p.

Ledo CAS, Silva SO \& Conceição KS (2003) Avaliação do coeficiente de variação na experimentação com bananeira. In: Simpósio brasileiro sobre bananicultura, Paracatu. Anais. Campo. 238-240

Lilliefors HW (1967) On the Kolmorov-Smirnov test for normality with mean and variance unknown. Journal of the American Statistical Association, 62:399-402.
Lima LL, Nunes GHS \& Bezerra Neto F (2004) Coeficientes de variação de algumas características do meloeiro: uma proposta de classificação. Horticultura Brasileira, 22:14-17.

Mohallem DF, Tavares M, Silva PL, Guimarães EC \& Freitas RF (2008) Avaliação do coeficiente de variação como medida de precisão em experimentos com frangos de corte. Arquivo Brasileiro de Medicina e Veterinária e Zootecnia, 60:449-453.

Ramalho MAP (2000) A experimentação em genética e melhoramento de plantas. Lavras, EDUFLA, 326p.

Rêgo ER, Rêgo MM, Silva DF, Cortez RM, Sapucay MJLC, Silva DR \& Silva Junior SJ (2009) Selection for leaf and plant size and longevity of ornamental peppers (Capsicum spp.) grown in greenhouse condition. Acta Horticulturae, 829:371-375.

Rêgo ER, Rêgo MM, Cruz CD, Finger FL \& Casali VWD (2010) Phenotypic diversity, correlation and importance of variables for fruit quality and yield traits in Brazilian peppers (Capsicum baccatum). Genetic Resources and Crop Evolution. 7:1-10

Sapucay MJLC, Araujo ER, Rego ER \& Rêgo MM (2009) Diversidade genética, importância relativa e correlação de caracteres quantitativos em pimenteiras. In: $49^{\circ}$ Congresso Brasileiro de Olericultura, Águas de Lindóia. Horticultura Brasileira. Brasília: ABH, 27:S1161-S1168.

Scapim CAS, Carvalho CGP \& Cruz CD (1995) Uma proposta de classificação dos coeficientes de variação para a cultura do milho. Pesquisa Agropecuária Brasileira, 30:683-686.

Snedecor GW \& Cochran WG (1980) Statistical methods. 7ed. Ames, The Iowa States University Press. 593p.

Storck, L, Garcia DC \& Lopes, SJ (2000) Experimentação vegetal. Santa Maria: UFSM. 198p. 